\title{
Hepatitis C Virus Mimics Effects of Glypican-3 on CD81 and Promotes Development of Hepatocellular Carcinomas via Activation of Hippo Pathway in Hepatocytes
}

Yuhua Xue, Wendy M. Mars, William Bowen, Aatur D. Singhi, John Stoops, and George K. Michalopoulos

From the Department of Pathology, University of Pittsburgh, Pittsburgh, Pennsylvania

Accepted for publication February 13, 2018.

Address correspondence to George K. Michalopoulos, M.D., Ph.D., Department of Pathology, University of Pittsburgh, 200 Lothrop St., BST S410, Pittsburgh, PA 15261. E-mail:

michalopoulosgk@upmc.edu.

\begin{abstract}
Glypican (GPC)-3 is overexpressed in hepatocellular carcinomas (HCCs). GPC3 binds to CD81. Forced expression of CD81 in a GPC3-expressing HCC cell line caused activation of Hippo, a decrease in ezrin phosphorylation, and a decrease in yes-associated protein (YAP). CD81 is also associated with hepatitis $C$ virus (HCV) entry into hepatocytes. Activation of CD81 by agonistic antibody causes activation of tyrosine-protein kinase SYK (SYK) and phosphorylation of ezrin, a regulator of the Hippo pathway. In cultures of normal hepatocytes, CD81 agonistic antibody led to enhanced phosphorylation of ezrin and an increase in nuclear YAP. HCV E2 protein mimicked GPC3 and led to enhanced Hippo activity and decreased YAP in cultured normal human hepatocytes. HCC tissue microarray revealed a lack of expression of CD81 in most HCCs, rendering them insusceptible to HCV infection. Activation of CD81 by agonistic antibody suppressed the Hippo pathway and increased nuclear YAP. HCV mimicked GPC3, causing Hippo activation and a decrease in YAP. HCV is thus likely to enhance hepatic neoplasia by acting as a promoter of growth of early CD81-negative neoplastic hepatocytes, which are resistant to HCV infection, and thus have a proliferative advantage to clonally expand as they participate in compensatory regeneration for the required maintenance of $100 \%$ of liver weight (hepatostat). (Am J Pathol 2018, 188: 1469-1477; https://doi.org/10.1016/j.ajpath.2018.02.013)
\end{abstract}

Hepatocellular carcinoma (HCC) has the fifth-leading solid tumor frequency in the world, and is the third-leading cause of cancer-related mortality. ${ }^{1}$ There is a growing trend of HCC incidence and mortality in middle-aged African American, Hispanic, and Caucasian populations in the United States. ${ }^{2}$ Aflatoxin contamination of food, hepatitis B virus, and hepatitis $\mathrm{C}$ virus (HCV) are prominent risk factors. ${ }^{3}$ The recent introduction of effective therapeutic agents against $\mathrm{HCV}$ provides hope for a future decrease in HCVassociated HCC. However, patients with established chronic HCV infection, and especially those with cirrhosis, are likely to continue to be a source for the development of HCC for many decades yet to come. Thus, investigation of pathways and molecules involved in HCC carcinogenesis, ${ }^{4,5}$ such as HCV-related effects on growth signaling pathways, ${ }^{6}$ might be helpful for early diagnosis and screening in high-risk patients, and for developing improved therapies for HCV-related HCCs.

Glypican (GPC)-3 is highly up-regulated in most $\mathrm{HCCs}^{7}$ but is expressed less in normal hepatocytes and nonmalignant liver lesions. ${ }^{8,9}$ It is encoded by a gene on the $\mathrm{X}$ chromosome. Loss-of-function mutation of GPC3 is associated with increased organ size (Simpson-Golabi-Behmel syndrome) and cancer formation. ${ }^{10}$ Transgenic expression of GPC3 in hepatocytes decreases both liver regeneration $^{11,12}$ and xenobiotic-induced coxsackievirus and adenovirus receptor (CAR) protein-mediated hepatomegaly. ${ }^{13}$ GPC3 binds to the Sonic hedgehog protein and also to

Supported by NIH/NIDDK Program Project grant P01 DK096690 (P.I David Perlmutter) (G.K.M.), the Cleveland Foundation, and the Menten Endowment Foundation of the University of Pittsburgh.

Disclosures: None declared. 
CD81, regulating the migration of hematopoietically expressed homeobox protein HHEX from the nucleus to CD81. ${ }^{14}$ As a heparin sulfate proteoglycan, GPC3 is associated with binding to and signaling of other proteins, ${ }^{15}$ such as wingless-type mouse mammary tumor virus integration site family (WNT), ${ }^{16}$ transforming growth factor $\beta,{ }^{17}$ and Sonic hedgehog. ${ }^{18}$

CD81 is the main binding protein for GPC $3{ }^{11,12,14}$ As a member of the tetraspanin protein family, CD81 transmits signals from two extracellular loops to induce cytoplasmic $\mathrm{N}$ - and C-terminal conformational changes. ${ }^{19}$ These conformational changes cause activation of the serine/threonine kinase SYK (tyrosine-protein kinase SYK), which in turn causes serine/threonine phosphorylation of linker protein ezrin. ${ }^{20}$ Previous studies have shown that ligation of CD81 with the agonistic antibody 5A6 causes phosphorylation of specifically and only ezrin. ${ }^{20}$ Phosphorylated (p)ezrin down-regulates the Hippo pathway, ${ }^{21}$ which in turn causes decreased phosphorylation and degradation of the yes-associated protein (YAP). Hepatocyte CD81, however, also mediates HCV entry into hepatocytes, along with two other proteins. ${ }^{22}$ Ezrin, a member of the ERM family (ezrin, radixin, and moesin), ${ }^{23}$ is up-regulated and correlates with malignant phenotype ${ }^{24}$ in HCC. Dysregulation of the Hippo pathway and elevated YAP activity have been shown in $50 \%$ of HCCs. ${ }^{5}$ The CD81-SYK-ezrin axis transmits signals that adjust cell morphology by cytoskeleton-protein interaction, and gene expression profile via YAP by downstream modulation of the Hippo pathway. Since GPC3 is a major binding ligand for CD81, in the present study we explored the interactions between GPC3 and CD81, and CD81 and $\mathrm{HCV}$ E2 protein in relation to regulation of the Hippo pathway and YAP protein in normal hepatocytes and HCC. HCV E2 protein mimicked the effects of GPC3 and caused activation of the Hippo pathway and a decrease in YAP, associated with a decrease in cell proliferation. Surprisingly, most HCCs, regardless of associated etiologies, do not express CD81. The absence of expression of CD81 suggests that loss of CD81 expression is an early, common event during neoplastic transformation of hepatocytes. The absence of CD81 would render early HCC resistant to HCV infection. ${ }^{25}$ In the case of HCV infection, and with HCV E2 protein causing down-regulation of YAP, randomly occurring CD81-negative hepatocytes would be resistant to $\mathrm{HCV}$ infection and thus likely to have a proliferation advantage in comparison to normal human hepatocytes. Because liver-to-body weight ratio is maintained by the proliferation of hepatocytes ${ }^{26}$ in situations of hepatocyte loss, ${ }^{27,28}$ CD81-negative hepatocytes are likely to clonally expand faster than $\mathrm{HCV}$-inhibited, CD81-positive normal hepatocytes. In the genotoxic environment of chronic inflammation, faster-proliferating, CD81-negative, and HCV-resistant hepatocytes are likely to accumulate genomic alterations leading to neoplasia. Thus, HCV would favor the expansion of such early HCC clones ${ }^{26}$ and act as a promoter of the formation of HCC. ${ }^{29}$

\section{Materials and Methods}

\section{Animal Protocols and Handling}

All procedures performed on mice were approved under the University of Pittsburgh Institutional Animal Care and Use Committee protocol 14053713, conducted according to NIH guidelines, ${ }^{30}$ and performed as described. At defined time points, the animals were sacrificed according to American Association for Accreditation of Laboratory Animal Care guidelines.

\section{Hepatoma Cell Lines, HepaRG Cell Line, Primary Rat Hepatocytes, Primary Human Hepatocytes}

Two rat hepatoma cell lines (JM1 and JM2) originally isolated from our laboratory ${ }^{31}$ were maintained in culture as in previous studies. ${ }^{32}$ HepaRG cells (Life Technologies, Grand Island, NY) are from a well-differentiated human HCC cell line often used a surrogate for normal human hepatocytes. HepaRG cells were cultured in Williams' Medium E (Life Technologies) plus HepaRG Thaw, Plate, General Purpose Medium Supplement (Life Technologies). Primary rat hepatocytes were isolated and maintained in culture as described previously. ${ }^{33}$ Primary human hepatocytes were kindly gifted from Dr. David Geller (University of Pittsburgh, Pittsburgh, PA). Primary rat hepatocytes and human hepatocytes were seeded into collagen-coated plates in minimum essential media for 2 hours, and changed to fresh hepatocyte growth medium for rat hepatocytes and hepatocyte maintenance medium (Lonza, Walkersville, MD) containing $10^{-7}$ dexamethasone, $5 \mu \mathrm{g} / \mathrm{mL}$ insulin, 5 $\mu \mathrm{g} / \mathrm{mL}$ transferrin, and $5 \mathrm{ng} / \mathrm{mL}$ sodium selenite for human hepatocytes.

\section{Transient Transfection of JM2 Cell Line and BrdU Incorporation Assay}

JM2 cells were transfected with pCMV6-Entry-CD81 overexpression plasmid (Origene, Rockville, MD) or pCMV6Entry plasmid as a control at 95\% confluency in 6-well plates using GenJet (SignaGen, Rockville, MD). Briefly, following the manufacturer's protocol, the cells were transfected with 1 $\mu \mathrm{g}$ of plasmid DNA in Dulbecco's modified Eagle's medium containing $10 \%$ fetal bovine serum overnight. Twelve-hour time point samples were collected next morning for total protein analysis. After 24 hours transfection of JM2 cells in 6-well plates at $50 \%$ confluency, $20 \mu \mathrm{L}$ of bromodeoxyuridine (BrdU) solution (Life Technologies) was added to the fresh medium. After 24 hours of incorporation, $2 \mathrm{~mL}$ of $10 \%$ formalin was used to fix these cells for 6 hours at room temperature. Plates were stained using an antibody specific to recognize BrdU (Life Technologies) by standard immunohistochemistry (IHC) protocol. The positive staining cells and total cells in the center of each well were counted under a normal light microscope. 


\section{Nuclear Protein Extraction}

Primary rat or human hepatocytes were washed two to three times with cold Hank's balanced salt solution (Corning Incorporated, Corning, NY) containing proteinase inhibitors. Scraped cells were collected into 1.5-mL Eppendorf tubes, centrifuged at $10 \times g$ to collect the pellet, and resuspended in $1 \times$ standard saline citrate, containing $5 \mathrm{mmol} / \mathrm{L}$ EDTA, $0.2 \%$ Nonidet P-40 (Sigma-Aldrich), $\mathrm{pH}$ 7.0, with proteinase inhibitors. The nuclei were examined under the microscope. They were isolated by centrifugation at $2.5 \times g$ for $10 \mathrm{mi}-$ nutes. The supernatant was removed, and used in the studies as the source of total cytoplasmic protein. The nuclei were resuspended twice with $1 \times$ standard saline citrate, containing $5 \mathrm{mmol} / \mathrm{L}$ EDTA, pH 7.0, with proteinase inhibitors. The resuspended nuclear pellet (in $1 \times$ standard saline citrate, 5 $\mathrm{mmol} / \mathrm{L}$ EDTA, $0.5 \%$ SDS with proteinase inhibitors) was used in the studies as total nuclear protein.

\section{Detection of Proteins by Western Blot Analysis}

Cell lysates were prepared as described. The protein expression levels of GPC3, CD81, YAP, p-YAP, SYK, $\beta$-actin, mammalian STE20-like protein kinase (MST)-1, p-MST1, serine/ threonine-protein kinase LATS (LATS) 1, p-LATS1-ezrin, and p-ezrin (Thr567) were evaluated by Western blot analysis. Forty micrograms of total protein extracted from the liver tissue or $20 \mu \mathrm{g}$ of total protein isolated from cells in culture was extracted with $10 \%$ sodium dodecyl sulfate-polyacrylamide for gel electrophoresis and transferred onto Immobilon-P membranes (Millipore, Bedford, MA). After transfer, membranes were stained with Ponceau $S$ to evaluate efficient loading and transfer of proteins. Blots were probed with primary antibody and secondary antibodies in Tris-buffered saline with Tween 20 containing 5\% bovine serum albumin (Sigma-Aldrich, St. Louis, MO). The following primary antibodies were used: anti-YAP, p-YAP, MST1, p-MST1, SYK, $\beta$-actin, LATS1 and p-LATS1, ezrin, and p-ezrin (Thr567) (Cell Signal Technologies, Danvers, MA), anti-GPC3 or CD81 (GeneTex, Irvine, CA). The membranes were processed with SuperSignal West Pico chemiluminescence substrate (Pierce, Rockford, IL) and exposed to X-ray films (Laboratory Product Sales, Rochester, NY).

\section{Tissue Microarrays and IHC Analysis}

Commercially available tissue microarrays were purchased from Biomax (Biomax Informatics AG, New Glarus, WI), and the IHC analysis protocols were described previously. ${ }^{32}$ Additional tissue microarrays of human HCC were also utilized from available material from the University of Pittsburgh pathology archives (constructed by A.D.S.). Mouse anti-human GPC3 monoclonal antibody (Santa Cruz, Dallas, TX) and mouse anti-human CD81 antibody (Santa Cruz) were used to detect the expression of Gpc3 and CD81. Sections $(4 \mu \mathrm{m})$ from paraffin-embedded liver tissue were stained with antibody against Yap (Cell Signal Technologies) using the avidin-biotin-peroxidase complex technique. The sections were counterstained with hematoxylin and eosin. The stained sections were imaged using an inverted microscope (Olympus, Center Valley, PA) at $\times 10$ and $\times 40$ for tissue microarrays. The data presented were merged from both of these arrays.

\section{Immunoprecipitation}

Five hundred micrograms of whole-cell lysates from regenerating liver were prepared in Tris-SDS lysis buffer and diluted to a final volume of $750 \mu \mathrm{L}$. Protein complexes were immunoprecipitated with $12 \mu \mathrm{g}$ of CD81 (Santa Cruz) or mouse IgG control (Santa Cruz) overnight at $4^{\circ} \mathrm{C}$ with end-over-end mixing followed by incubation with protein $\mathrm{A} /$ $\mathrm{G}$ beads (Santa Cruz). Complexes were centrifuged at $5.5 \times g$ for 5 minutes and washed three times in phosphate-buffered saline buffer before resuspension in $4 \times$ Laemmli loading buffer. Immunoprecipitated protein complexes were separated and probed by Western blot analysis.

\section{Statistical Analysis}

Tissue array data were analyzed using Prism software version 5 (GraphPad Software, San Diego, CA). $P<0.05$ was considered statistically significant.

\section{Results}

Agonistic Anti-CD81 Antibody (5A6) Enhances Levels of Nuclear Yap Protein in Primary Rat Hepatocytes

To investigate the effect of GPC3-CD81-ezrin axis in normal hepatocytes, the agonistic anti-CD81 antibody 5A6 was first examined in primary rat hepatocytes. This antibody has been shown in previous studies to ligate with CD81 and specifically activate only ezrin phosphorylation via SYK. ${ }^{20}$ To preclude a false-positive effect of CD81 agonistic antibody (5A6), ${ }^{20}$ heat-inactivated $5 \mathrm{~A} 6$ antibody at $100^{\circ} \mathrm{C}$ for a half-hour was used as control. Five hours of treatment with $5 \mathrm{~A} 6$ at a concentration of $1 \mu \mathrm{g} / \mathrm{mL}$ of cultures once daily for 6 days led to enhanced Yap nuclear translocation (Figure 1) and an increase in ezrin phosphorylation (Thr567). There was no change in total ezrin (Figure 1).

\section{Forced Expression of CD81 in Glypican-3-Expressing Hepatoma Cell Line JM2 Leads to Decreased Phosphorylation of Ezrin, Increased Activation of Components of the Hippo Pathway, Decreased Nuclear Yap, and Decreased Cell Proliferation}

The JM2 rat hepatoma cell line has very little CD81 protein expression but expresses high levels of Gpc3. Therefore, this cell line was used to study the effect of forced CD81 expression, in the presence of Gpc3, on the Hippo pathway, 


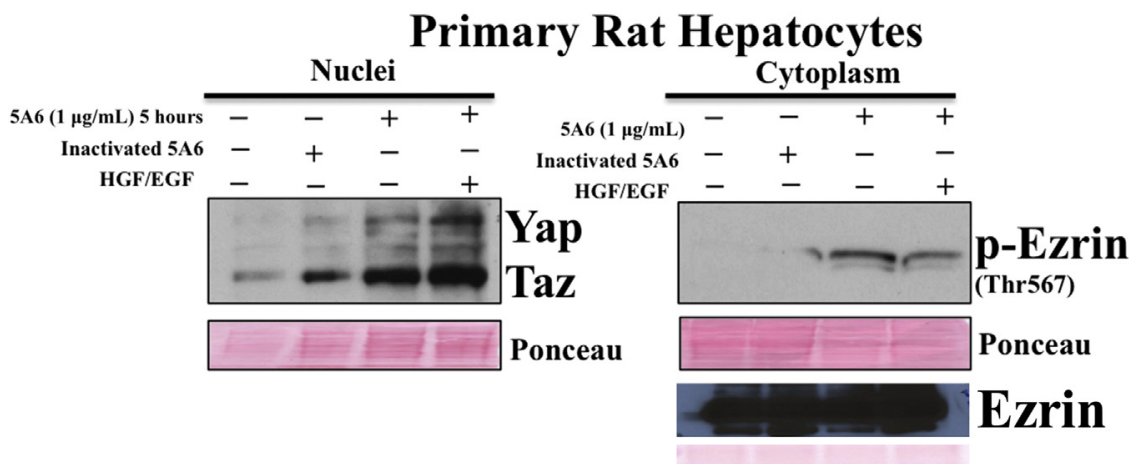

Nuclear Yap

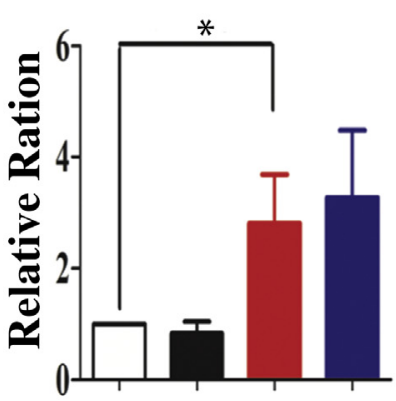

Cyto p-ezrin

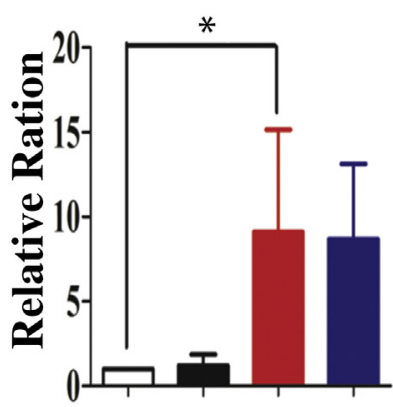

Figure 1 Effects of the CD81 agonistic antibody $5 \mathrm{~A} 6$ on hepatocyte proliferation. Rat hepatocytes at day 6 in primary culture were stimulated with $1 \mu \mathrm{g} / \mathrm{mL}$ of $5 \mathrm{~A} 6$ or inactivated control for 5 hours. Yes-associated protein (Yap) and tafazzin (Taz) expression levels in nuclear extracts (left panels), total ezrin and phosphorylated (p)-ezrin (Thr567) expression levels in cytoplasm (right panels) were detected by Western blot analysis. Each bar indicates the ratio between the intensity of the relative band divided by the intensity of the Ponceau stain. The bars in the graphs in the bottom panels correspond to the labels and conditions shown in the corresponding top panels for the Western gels (white bars, control medium; black bars, inactivated $5 \mathrm{~A} 6$ agonistic antibody; red bars, $5 \mathrm{~A} 6$ agonistic antibody; blue bars, $5 \mathrm{~A} 6$ agonistic antibody+HGF). Data are expressed as means \pm SEM of four separate cultures. ${ }^{*} P<0.05$. HGF/EGF, hepatocyte growth factor/epidermal growth factor.
Yap, ezrin, and p-ezrin. After transient transfection of JM2 cells with CD81 cDNA for 12 hours, a significant increase of CD81 expression was observed (Figure 2A). Gpc3 was prominently expressed and its expression was not affected by CD81 expression. In the presence of both CD81 and Gpc3 (Figure 2A), however, there was decrease in levels of p-ezrin (Thr567) (Figure 2A), an increase in cleaved and activated p-Mst1 (first kinase component of the Hippo pathway), a decrease in Yap, and an increase in $\mathrm{p}$-Yap (Figure 2B). There was no change in total ezrin. As expected from the decrease in Yap, there was also concomitant decrease in the Yap-controlled protein connective tissue growth factor $^{34}$ (Supplemental Figure S1). Furthermore, CD81 expression in the Gpc3-expressing cells caused a substantial decrease in cell proliferation (Figure 2C). This in vitro CD81-regain experiment suggests that CD81 expression in hepatoma cells in the presence of GPC3 would negatively regulate levels of nuclear YAP in HCC cells, and that a loss of CD81 in the presence of GPC3 would potentially confer to HCC cells a growth advantage.
A

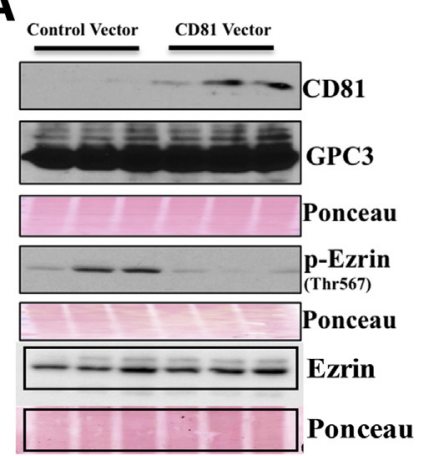

B

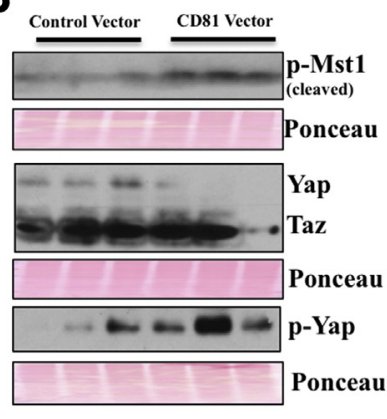

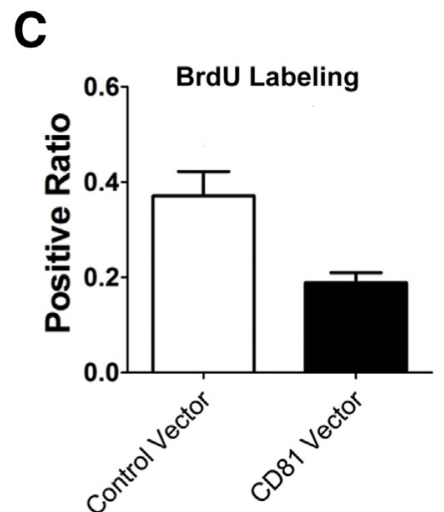

Figure 2 Forced expression of CD81 in glypican (Gpc)-3-expressing JM2 hepatoma cells results in activation of the Hippo pathway, a decrease in nuclear Yap, and a decrease in the percentage of bromodeoxyuridine (BrdU)-positive nuclei. JM2 cells were transiently transfected with pCMV6-CD81 plasmid, and after 12 hours, total cell lysates were collected for protein expression analysis. A: Expression of CD81, Gpc3, ezrin, and phosphorylated (p)-ezrin (Thr567). B: Cleaved (activated) p-mammalian STE20-like protein kinase (Mst)-1, yes-associated protein (Yap), and p-Yap. In both $\mathbf{A}$ and $\mathbf{B}$, each band represents a separate cell culture. Results are representative of four experiments. C: JM2 cells in sparse density (50\%) after 24 hours of transfection and 24 hours of BrdU incorporation. Positive ratio indicates the fraction of cells whose nuclei were positive for BrdU by IHC analysis. Data are expressed as means \pm SEM, derived from four separate cultures for each category (C). $P=0.0078$. 


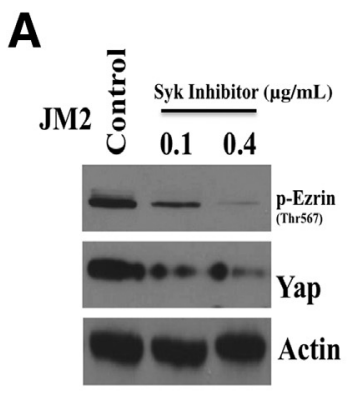

B
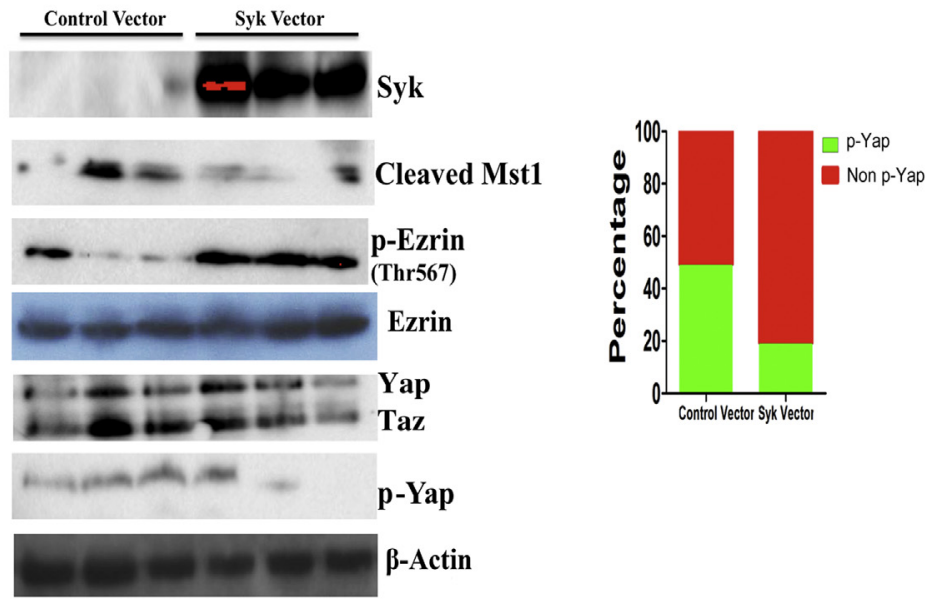

Figure 3 Inhibition of CD81-associated tyrosine-protein kinase SYK (Syk) results in decrease in yes-associated protein (Yap) expression and ezrin phosphorylation. A: JM2 rat hepatocellular carcinoma cells were treated with Syk inhibitor at 0.1 and $0.4 \mu \mathrm{g} / \mathrm{mL}$ for 5 hours, and total proteins were analyzed by Western blot for Yap, ezrin, and phosphorylated (p)-ezrin (Thr567). B: JM2 cells were transiently transfected with pCMV6-Syk plasmid, and after 12 hours, total cell lysates were collected for protein expression analysis of Syk, ezrin, and p-ezrin (Thr567), cleaved p-mammalian STE20-like protein kinase (Mst)-1, Yap/tafazzin (Taz), and p-Yap. Red indicates high saturation of the band. C: Quantitative analysis of relative percentage of p-Yap and Yap expression from C.

\section{Syk Increases Yap Activity by Modulating Phosphorylation of Ezrin}

Since the effects of CD81 are mediated via Syk, the roles of Syk in ezrin phosphorylation and regulation of Hippo and Yap were investigated. The effects of inhibition of Syk were studied using a specific Syk inhibitor (catalog number PRT062607; Selleck Chemicals, Houston, TX). There was dose-dependent decrease in p-ezrin in JM2 cells (Figure 3A), and less Yap activity. Alternatively, after transient transfection of JM2 cells with a vector expressing Syk cDNA for 12 hours, a significant increase of Syk expression was observed (Figure 3B). There was a concomitant decrease in levels of activated (cleaved) Mst1, an increase in p-ezrin, and a decrease in p-Yap (Figure 3B), the latter indicating down-regulation of the Hippo pathway. There was no change in total ezrin. Syk overexpression did not result in an increase in Yap per se, but it caused a dramatic reduction in $p$-Yap, demonstrating the severe down-regulation of the Hippo pathway. The relative proportions of Yap to p-Yap are shown in Figure 3C.

\section{HCV E2, p-Ezrin, Hippo Components, and YAP in} Human Hepatocytes

In addition to GPC3, the $\mathrm{E} 2$ protein of $\mathrm{HCV}$ is also known to interact with CD81. CD81 interaction with $\mathrm{HCV}$ is involved in HCV entry into hepatocytes, and it is mediated by the interaction of CD81 with HCV envelope protein E2. ${ }^{22}$ Since CD81 plays a role in the regulation of the Hippo pathway and YAP, the effects of HCV E2 protein in human HCC HepaRG cells and normal human hepatocytes in primary culture on the CD81/Hippo/YAP signaling pathway were explored
(Figure 4). HepaRG is a well-differentiated human HCC cell line (Thermo Fisher Scientific, Pittsburgh, PA) that expresses CD81 (Figure 4) and mimics many of the properties of normal human hepatocytes. ${ }^{35}$ The results of treatment of HepaRG cells with HCV E2 protein are shown in Figure 4A. After 5 hours of HCV E2 protein treatment $(10 \mu \mathrm{g} / \mathrm{mL})$, HepaRG cells had lower levels of nuclear YAP. E2 protein treatment also led to an increased activation of LATS1 (p-LATS1), the second kinase component of the Hippo pathway. MPS one binder kinase activator-like protein (MOB)-1 is a known target of MST1/2. An increase in the proportion of p-MOB1 (Thr35) is a surrogate marker for enhanced activity of MST1/ $2^{36}$ (the first kinase of the Hippo pathway). Next, E2 protein was used to treat primary human hepatocytes (Figure 4B). Treatment with E2 protein caused a decrease in nuclear YAP and p-ezrin (Thr567) and an increase in activated (phosphorylated) MST1. This was found with cultures of hepatocytes from three separate donors. Treatment with HCV E2 protein did not have any effect on the levels of CD81 itself or total ezrin (data not shown), in either HepaRG or primary human hepatocytes. The overall results demonstrate that the HCV E2 protein interacting with the CD81 mimics the effects of GPC 3 and results in decreased p-ezrin, enhanced activation of the MST1 and LATS1 kinases of the Hippo pathway, and decrease in nuclear YAP. There was no change in total ezrin.

\section{Down-Regulation of CD81 in Human HCC}

GPC3 and CD81 expression profile in human HCC samples were evaluated using a commercial HCC tissue microarray (Biochain Institute Inc., Newark, CA) as well as constructed HCC tissue microarrays from the surgical pathology archives of the University of Pittsburgh. The results of the studies from 


\section{A}

HepaRG Total Cell Lystate

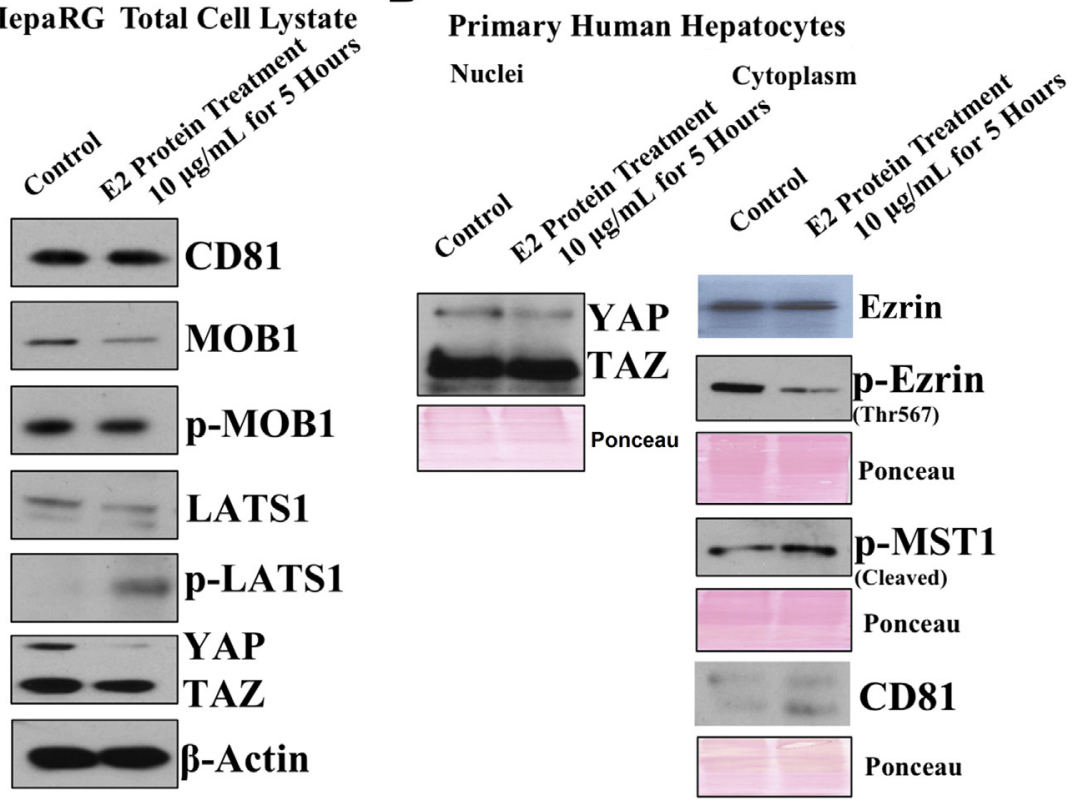

B
Figure 4 Hepatitis $\mathrm{C}$ virus (HCV) E2 protein enhances Hippo activity and decreases yesassociated protein (Yap) in human hepatocellular carcinoma and primary human hepatocytes. A: HepaRG cells were treated with HCV E2 protein 10 $\mu \mathrm{g} / \mathrm{mL}$ for 5 hours, and whole-cell lysates were analyzed by Western blot for CD81, MPS one binder kinase activator-like protein (Mob)-1, phosphorylated ( $p)-M 0 B 1$, serine/threonine-protein kinase LATS-1, p-LATS1, and YAP/tafazzin (Taz). B: Primary human hepatocytes were treated with $10 \mu \mathrm{g} /$ $\mathrm{mL}$ HCV E2 protein for 5 hours, and total nuclear protein and cytoplasmic fraction were analyzed separately by Western blot for YAP, CD81, ezrin, and p-ezrin (Thr567) and cleaved (activated) $\mathrm{p}$-mammalian STE20-like protein kinase (MST)-1. the two microarrays were combined. The expression levels of GPC3 and CD81 were investigated in 310 sections from 155 primary HCC by IHC analysis (Figure 5). Immunoreactivity of GPC3 was observed in the cell membrane and extracellular matrix of both tumor cells and normal cells. There was much higher immunoreactivity in $\mathrm{HCC}$ samples compared to normal liver. Immunoreactivity of CD81 protein was not detectable in most HCCs but was detectable in the cell membrane of associated normal cells in the tumor stroma. Eighty-nine percent (275/310) of HCC samples stained positive for GPC3. In contrast, only 22\% (69/310) of HCC tissues showed plasma membrane immunoreactivity for CD81 (Table 1). These findings demonstrate that most HCCs do not express CD81.

\section{Discussion}

Evidence in other tissues from the combined literature and work in mouse liver from our laboratory have established that CD81, a tetraspanin, is associated with signaling
A

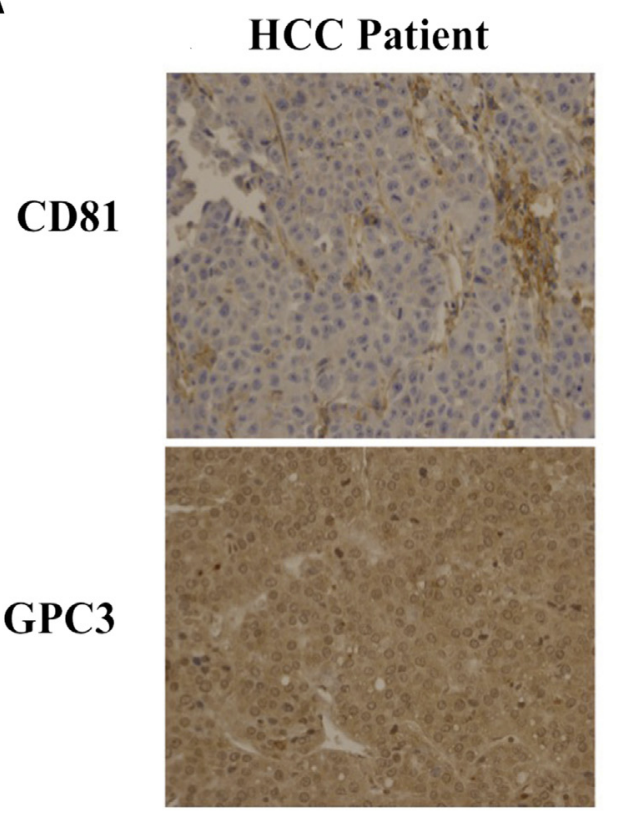

B

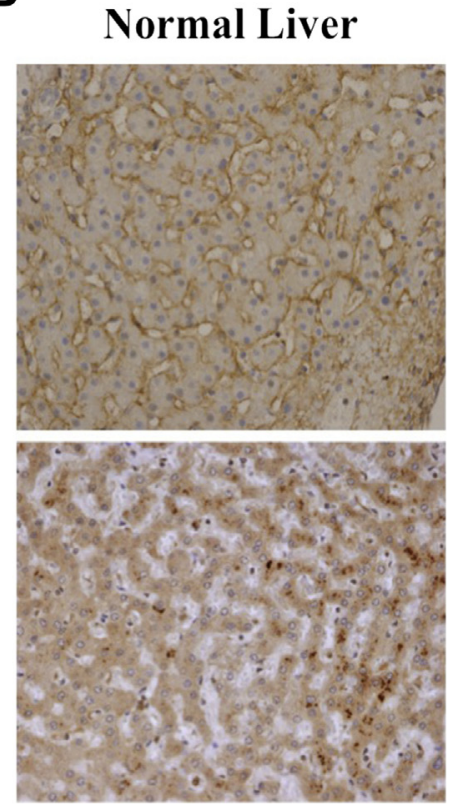

Figure 5 Plasma membrane CD81 expression is absent in most human hepatocellular carcinomas (HCCs). Human HCC tissue arrays were used to evaluate the expression pattern of CD81 and glypican (GPC)-3. A total of 310 sections of primary HCC from HCC patients using tissue microarrays (Materials and Methods) were analyzed by IHC staining. A: Representative images of CD81 and GPC3 staining in HCC. B: Representative pictures of CD81 and GPC3 staining in normal liver. Original magnification, $\times 400$. 
Table 1 CD81 and GPC3 Staining Results of HCC Patients and Normal Tissues in Tissue Array LV482 and HCC Tissues in Tissue Arrays Constructed by the Department of Pathology, University of Pittsburgh

\begin{tabular}{|c|c|c|c|c|c|c|}
\hline \multirow[b]{2}{*}{ Type of tissue } & \multicolumn{3}{|c|}{$\operatorname{CD} 81(n=310)$} & \multicolumn{3}{|c|}{ GPC3 $(n=310)$} \\
\hline & Negative & Positive & $P$ & Negative & Positive & $P$ \\
\hline $\mathrm{HCC}, n(\%)$ & $241(78)$ & $69(22)$ & $<0.0001$ & $35(11)$ & $275(89)$ & 0.6053 \\
\hline Normal & 0 & 8 & & 0 & 8 & \\
\hline
\end{tabular}

Fisher exact test, and $\chi^{2}$ test for all of the other analyses.

GPC, glypican; HCC, hepatocellular carcinoma.

capability, regulated by GPC $3^{13,14}$ and mediated in total, or in part, by SYK. ${ }^{20}$ This CD81-SYK interaction affects, among other targets, the Hippo pathway ${ }^{13}$ and levels of nuclear YAP as well Tyr-phosphorylation of ezrin. ${ }^{22}$ Signaling functions of CD81 were first demonstrated by treatment with the specific agonistic antibody 5A6. ${ }^{20}$ The purpose of this study was to highlight the importance of the CD81 signaling in hepatocyte growth biology, after the discovery of the interaction with GPC 3 and the fact that mice with transgenic expression of Gpc3 in hepatocytes have a decrease in Yap and decreased liver regeneration. ${ }^{12,13}$ However, in view of the association of CD81 with the early steps of HCV infection and also the association of the lack of $\mathrm{CD} 81$ with resistance to infection by $\mathrm{HCV},{ }^{25}$ these results are also of relevance in offering an explanation for the strong causal association between HCC development and chronic HCV infection. The current work provides evidence that HCV E2 protein mimics GPC3 and results in the activation of the Hippo pathway and decreased expression of YAP. The decrease in YAP expression resulting from HCV infection is likely to have an inhibitory effect on hepatocyte growth, affecting all hepatocytes infected with $\mathrm{HCV}$. It is well established that normal polyploid human and rodent hepatocytes are typically aneuploid and have random loss of chromosomes. ${ }^{37,38}$ In any chronic hepatic inflammation involving loss of hepatocytes, there is a ploidy reversal with increasing numbers of diploid/aneuploid cells. ${ }^{39}$ Thus, recessive genomic alterations obtained during persistent proliferation in the genotoxic environment of chronic inflammation ${ }^{40}$ are often likely to be expressed due to allelic imbalance. Since loss of CD81 expression is seen in most HCCs regardless of associated etiology, it is safe to speculate that a loss of CD81 expression is an early common event in the development of hepatic neoplasia. In chronic HCV infection, the HCV E2 protein interacting with CD81 would negatively affect the growth of normal, CD81-expressing hepatocytes. The decrease in YAP expression should be associated with decreased growth of CD81-expressing normal human hepatocytes and a decreased ability to participate in maintenance of liver mass (hepatostat). ${ }^{26}$ On the other hand, any hepatocytes that have lost CD81 expression are known to be resistant to $\mathrm{HCV}$ infection $^{25}$ and thus will be capable of expanding into enhanced clonal growth faster than the normal hepatocytes and make an enhanced contribution in compensating for the loss of hepatocytes caused by HCV infection. In that regard, they have an advantage over the CD81-expressing, HCV infection-prone, normal hepatocytes. In the context of HCV infection inhibiting growth of normal hepatocytes by suppressing YAP signaling, early CD81-negative HCC cells resistant to HCV infection would continue to expand, ${ }^{41}$ mimicking the earlier model of the resistant hepatocyte of liver cancer growth, first described by Farber et al. ${ }^{29}$ In this context, HCV would be enhancing hepatic neoplasia not directly, but by acting as a tumor promoter, creating a growth advantage for early neoplastic, CD81-negative hepatocytes. Since HCV does not integrate into DNA and does not contain activating oncogenes, the proposed mechanism described earlier in this paragraph might be crucial to understanding the relation of HCV to liver carcinogenesis. CD81 expression, if properly induced, may also carry additional therapeutic opportunities for HCC. Of interest, CD81 was absent in $78 \%$ of HCC in the tumor microarray study (Table 1); another study demonstrated the presence of $\mathrm{HCV}$ antigens in only $28 \%$ of HCCs in cases of HCV infection, ${ }^{39}$ a ratio that approximates $22 \%$ CD81-positive HCCs in this study.

Our study also highlights an incompatibility between the expression of high levels of both CD81 and GPC3 in the same cells (Figure 2). Undoubtedly a joint CD81 and GPC3 expression is possible if other pathways were also to be altered, rendering such interaction less harmful. It is of interest, however, that overexpression of CD81 in GPC3expressing HCC cell lines resulted in a decrease in cell replication, activation of the Hippo pathway components, and a decrease in Yap. Increased expression of Yap is a common feature in HCC, and it is likely that combined expression of high levels of GPC 3 and CD81 would cause a decrease in YAP and thus not be compatible with a high rate of HCC growth, whereas high levels of expression of GPC3 in the absence of expression of CD81 would not limit neoplastic growth. This indeed seems to be the case, as shown in Figures 2 and 5. At present, there is no mechanistic correlation between CD81 and direct regulation of GPC3. Thus, it is likely that this combination is a result of natural selection in the context of the milieu of the development of the early neoplasms. Supporting this hypothesis is also the fact that the absence of CD81 in the plasma membrane of HCC in the tissue arrays is seen not only in chronic HCV infection but also in HCC associated with other hepatic diseases. Recent studies have also shown that GPC3 by itself, in the absence of CD81, may actually 
have enhancing effects on HCC growth by interacting with the WNT and Hippo pathways. ${ }^{16}$

\section{Acknowledgment}

We thank Dr. David Geller (University of Pittsburgh, Pittsburgh, PA) for primary human hepatocytes.

\section{Supplemental Data}

Supplemental material for this article can be found at https://doi.org/10.1016/j.ajpath.2018.02.013.

\section{References}

1. Forner A, Llovet JM, Bruix J: Hepatocellular carcinoma. Lancet 2012, 379:1245-1255

2. Altekruse SF, McGlynn KA, Reichman ME: Hepatocellular carcinoma incidence, mortality, and survival trends in the United States from 1975 to 2005. J Clin Oncol 2009, 27:1485-1491

3. Bouchard MJ, Navas-Martin S: Hepatitis B and C virus hepatocarcinogenesis: lessons learned and future challenges. Cancer Lett 2011, 305:123-143

4. Perra A, Kowalik MA, Ghiso E, Ledda-Columbano GM, Di Tommaso L, Angioni MM, Raschioni C, Testore E, Roncalli M, Giordano S, Columbano A: YAP activation is an early event and a potential therapeutic target in liver cancer development. J Hepatol 2014, 61:1088-1096

5. Johnson R, Halder G: The two faces of Hippo: targeting the Hippo pathway for regenerative medicine and cancer treatment. Nat Rev Drug Discov 2014, 13:63-79

6. Wang SK, Zynger DL, Hes O, Yang XJ: Discovery and diagnostic value of a novel oncofetal protein: glypican 3. Adv Anat Pathol 2014 $21: 450-460$

7. Filmus J, Capurro M: Glypican-3: a marker and a therapeutic target in hepatocellular carcinoma. FEBS J 2013, 280:2471-2476

8. Capurro M, Wanless IR, Sherman M, Deboer G, Shi W, Miyoshi E, Filmus J: Glypican-3: a novel serum and histochemical marker for hepatocellular carcinoma. Gastroenterology 2003, 125:89-97

9. Libbrecht L, Severi T, Cassiman D, Vander Borght S, Pirenne J, Nevens F, Verslype C, van Pelt J, Roskams T: Glypican-3 expression distinguishes small hepatocellular carcinomas from cirrhosis, dysplastic nodules, and focal nodular hyperplasia-like nodules. Am J Surg Pathol 2006, 30:1405-1411

10. Kim H, Xu GL, Borczuk AC, Busch S, Filmus J, Capurro M, Brody JS, Lange J, D'Armiento JM, Rothman PB, Powell CA: The heparan sulfate proteoglycan GPC3 is a potential lung tumor suppressor. Am J Respir Cell Mol Biol 2003, 29:694-701

11. Liu B, Bell AW, Paranjpe S, Bowen WC, Khillan JS, Luo JH, Mars WM, Michalopoulos GK: Suppression of liver regeneration and hepatocyte proliferation in hepatocyte-targeted glypican 3 transgenic mice. Hepatology 2010, 52:1060-1067

12. Liu B, Paranjpe S, Bowen WC, Bell AW, Luo JH, Yu YP, Mars WM, Michalopoulos GK: Investigation of the role of glypican 3 in liver regeneration and hepatocyte proliferation. Am J Pathol 2009, 175: $717-724$

13. Lin CW, Mars WM, Paranjpe S, Donthamsetty S, Bhave VS, Kang LI, Orr A, Bowen WC, Bell AW, Michalopoulos GK: Hepatocyte proliferation and hepatomegaly induced by phenobarbital and 1,4-bis [2(3,5-dichloropyridyloxy)] benzene is suppressed in hepatocyte-targeted glypican 3 transgenic mice. Hepatology 2011, 54:620-630
14. Bhave VS, Mars W, Donthamsetty S, Zhang X, Tan L, Luo J, Bowen WC, Michalopoulos GK: Regulation of liver growth by glypican 3, CD81, hedgehog, and Hhex. Am J Pathol 2013, 183:153-159

15. De Cat B, David G: Developmental roles of the glypicans. Semin Cell Dev Biol 2001, 12:117-125

16. Gao W, Kim H, Feng M, Phung Y, Xavier CP, Rubin JS, Ho M: Inactivation of Wnt signaling by a human antibody that recognizes the heparan sulfate chains of glypican-3 for liver cancer therapy. Hepatology 2014, 60:576-587

17. Sun CK, Chua MS, He J, So SK: Suppression of glypican 3 inhibits growth of hepatocellular carcinoma cells through up-regulation of TGF-beta2. Neoplasia 2011, 13:735-747

18. Capurro MI, Xu P, Shi W, Li F, Jia A, Filmus J: Glypican-3 inhibits Hedgehog signaling during development by competing with patched for Hedgehog binding. Dev Cell 2008, 14:700-711

19. Huang S, Tian H, Chen Z, Yu T, Xu A: The evolution of vertebrate tetraspanins: gene loss, retention, and massive positive selection after whole genome duplications. BMC Evol Biol 2010, 10:306

20. Coffey GP, Rajapaksa R, Liu R, Sharpe O, Kuo CC, Krauss SW, Sagi Y, Davis RE, Staudt LM, Sharman JP, Robinson WH, Levy S: Engagement of CD81 induces ezrin tyrosine phosphorylation and its cellular redistribution with filamentous actin. J Cell Sci 2009, 122: 3137-3144

21. Angus L, Moleirinho S, Herron L, Sinha A, Zhang X, Niestrata M, Dholakia K, Prystowsky MB, Harvey KF, Reynolds PA, GunnMoore FJ: Willin/FRMD6 expression activates the Hippo signaling pathway kinases in mammals and antagonizes oncogenic YAP. Oncogene 2012, 31:238-250

22. Bukong TN, Kodys K, Szabo G: Human ezrin-moesin-radixin proteins modulate hepatitis C virus infection. Hepatology 2013, 58: $1569-1579$

23. Mangeat P, Roy C, Martin M: ERM proteins in cell adhesion and membrane dynamics. Trends Cell Biol 1999, 9:187-192

24. Lee JS, Heo J, Libbrecht L, Chu IS, Kaposi-Novak P, Calvisi DF, Mikaelyan A, Roberts LR, Demetris AJ, Sun Z, Nevens F, Roskams T, Thorgeirsson SS: A novel prognostic subtype of human hepatocellular carcinoma derived from hepatic progenitor cells. Nat Med 2006, 12 : $410-416$

25. Zhang YY, Zhang BH, Ishii K, Liang TJ: Novel function of CD81 in controlling hepatitis C virus replication. J Virol 2010, 84:3396-3407

26. Michalopoulos GK: Hepatostat: liver regeneration and normal liver tissue maintenance. Hepatology 2017, 65:1384-1392

27. Michalopoulos GK: Principles of liver regeneration and growth homeostasis. Compr Physiol 2013, 3:485-513

28. Michalopoulos GK, DeFrances MC: Liver regeneration. Science 1997, 276:60-66

29. Farber E, Parker S, Gruenstein M: The resistance of putative premalignant liver cell populations, hyperplastic nodules, to the acute cytotoxic effects of some hepatocarcinogens. Cancer Res 1976, 36:3879-3887

30. Committee for the Update of the Guide for the Care and Use of Laboratory Animals; National Research Council: Guide for the Care and Use of Laboratory Animals. Eighth Edition. Washington, DC, National Academies Press, 2011

31. Novicki DL, Jirtle RL, Michalopoulos G: Establishment of two rat hepatoma cell strains produced by a carcinogen initiation, phenobarbital promotion protocol. In Vitro 1983, 19:191-202

32. Koral K, Paranjpe S, Bowen WC, Mars W, Luo J, Michalopoulos GK: Leukocyte-specific protein 1: a novel regulator of hepatocellular proliferation and migration deleted in human hepatocellular carcinoma. Hepatology 2015, 61:537-547

33. Bhave VS, Paranjpe S, Bowen WC, Donthamsetty S, Bell AW, Khillan JS, Michalopoulos GK: Genes inducing iPS phenotype play a role in hepatocyte survival and proliferation in vitro and liver regeneration in vivo. Hepatology 2011, 54:1360-1370

34. Shimomura T, Miyamura N, Hata S, Miura R, Hirayama J, Nishina H The PDZ-binding motif of Yes-associated protein is required for its co-activation of TEAD-mediated CTGF transcription and oncogenic 
cell transforming activity. Biochem Biophys Res Commun 2014, 443: 917-923

35. Andersson TB, Kanebratt KP, Kenna JG: The HepaRG cell line: a unique in vitro tool for understanding drug metabolism and toxicology in human. Expert Opin Drug Metab Toxicol 2012, 8: 909-920

36. Zeng Q, Hong W: The emerging role of the hippo pathway in cell contact inhibition, organ size control, and cancer development in mammals. Cancer Cell 2008, 13:188-192

37. Duncan AW, Hanlon Newell AE, Bi W, Finegold MJ, Olson SB, Beaudet AL, Grompe M: Aneuploidy as a mechanism for stressinduced liver adaptation. J Clin Invest 2012, 122:3307-3315

38. Duncan AW, Hanlon Newell AE, Smith L, Wilson EM, Olson SB, Thayer MJ, Strom SC, Grompe M: Frequent aneuploidy among normal human hepatocytes. Gastroenterology 2012, $142: 25-28$

39. Anti M, Marra G, Rapaccini GL, Rumi C, Bussa S, Fadda G, Vecchio FM, Valenti A, Percesepe A, Pompili M, Armelao F, Gentiloni N: DNA ploidy pattern in human chronic liver diseases and hepatic nodular lesions. Flow cytometric analysis on echo-guided needle liver biopsy. Cancer 1994, 73:281-288

40. Ioannidou A, Goulielmaki E, Garinis GA: DNA damage: from chronic inflammation to age-related deterioration. Front Genet 2016, 7:187

41. Rullier A, Trimoulet P, Urbaniak R, Winnock M, Zauli D, Ballardini G, Rosenbaum J, Balabaud C, Bioulac-Sage P, Le Bail B: Immunohistochemical detection of HCV in cirrhosis, dysplastic nodules, and hepatocellular carcinomas with parallel-tissue quantitative RT-PCR. Mod Pathol 2001, 14:496-505 